\title{
NEGATIVE INTERFERENCE AND THE USE OF FLANKING MARKERS IN FINE-STRUCTURE MAPPING IN FUNGI
}

\author{
J. R. S. FINCHAM \\ Department of Genetics, University of Leeds, Leeds LS2 9JT
}

Received 21.iii.74

\section{SUMMARY}

\begin{abstract}
Mapping within the am gene of Neurospora crassa is possible only because of a marked polarity of conversion within the gene, seen in the unequal frequencies of the two parental flanking marker combinations among the selected intragenic recombinants. The conventional comparison of the frequencies of the two flanking marker recombinant classes gives only uncertain and conflicting indications of the orientation of sites within the gene. While other possible interpretations are not ruled out, it is suggested that one relatively simple way of explaining the data is to suppose (a) that most recombination within am is due to conversion without immediately associated crossing-over, and $(b)$ that recombinational events (conversions and cross-overs) tend to occur in tight clusters, so that conversion within the gene has a high probability (apparently about 25 per cent in am) of being accompanied by an independently initiated cross-over close by and on either side more or less indiscriminately.
\end{abstract}

THE conventional method of mapping within genes using random meiotic products is to analyse crosses of the form

$$
P a_{1} a_{2}^{+} D \times p a_{1}^{+} a_{2} d
$$

where $a_{1}$ and $a_{2}$ are different mutant sites within a gene and $p$ and $d$ are flanking markers, proximal and distal respectively, in relation to the centromere. Where $a_{1}$ and $a_{2}$ are auxotrophic mutants, as is usually the case in fungi, selection is made for prototrophs $\left(a_{1}^{+} a_{2}^{+}\right)$under conditions which are unselective with respect to the flanking markers.

It is convenient to designate the four flanking marker combinations as follows:

$$
\begin{aligned}
& P D=\mathrm{P} 1 \quad \begin{array}{l}
\text { (parental combination originally associated with the proximal } \\
\text { site } \left.a_{1}\right) .
\end{array} \\
& p d=\mathrm{P} 2 \begin{array}{l}
\text { (parental combination originally associated with the distal } \\
\text { site } \left.a_{2}\right) .
\end{array} \\
& p D=\mathrm{R} 1 \begin{array}{l}
\text { (the recombinant class expected to result from a single cross- } \\
\text { over between } \left.a_{1} \text { and } a_{2}\right) .
\end{array} \\
& P d=\mathrm{R} 2 \begin{array}{l}
\text { (requires a triple cross-over on a conventional crossing-over } \\
\text { interpretation). }
\end{array}
\end{aligned}
$$

It is, of course, necessary to exclude the possibility that selection acting on the flanking markers is affecting the relative frequencies of the four recovered classes. No such selective effects appear to operate on the flanking markers used in the studies discussed here (Fincham, 1967; Smyth, 1971).

The conventional criterion for determining site order within the gene, and the orientation of this order with respect to the flanking markers, is the relationship $\mathrm{R} 1 \gg \mathrm{R} 2$. This criterion was originally based on the classical cross-over theory. On this theory, Rl would be a single- and R2 a triplecross-over class. However, it is now abundantly clear both in fungi (Fincham 
and Day, 1971) and Drosophila (Chovnick et al., 1971) that intragenic recombination is most usually due not to reciprocal crossing-over as traditionally conceived, but to non-reciprocal information transfer from one chromatid to its homologue, a process known as gene conversions. Currently favoured models for recombination (Holliday, 1968; Meselson and Radding, personal communication) regard conversion and crossing-over as consequences of the same primary process; the primary event (hybrid DNA formation leading to gene conversion) is thought to bring about crossing-over in a proportion of cases (about 50 per cent in yeast-Hurst et al., 1972) and, conversely, some degree of non-reciprocal transfer or copying of DNA is expected to occur in the immediate vicinity of all cross-overs. By a happy coincidence, this revised view of the nature of intragenic recombination also leads to the $\mathrm{R} 1>>\mathrm{R} 2$ prediction, since the $\mathrm{R} 1$ class will result whenever either $a_{1}$ or $a_{2}$ is converted and a cross-over is formed at or immediately adjacent to the converted segment as a consequence of the same primary event (lower half of fig. 1). Without complicating the model one can only explain the $\mathrm{R} 2$ class as a result of conversion at one site accompanied, as a separate event, by a cross-over on the far side of the (non-converted) second site.

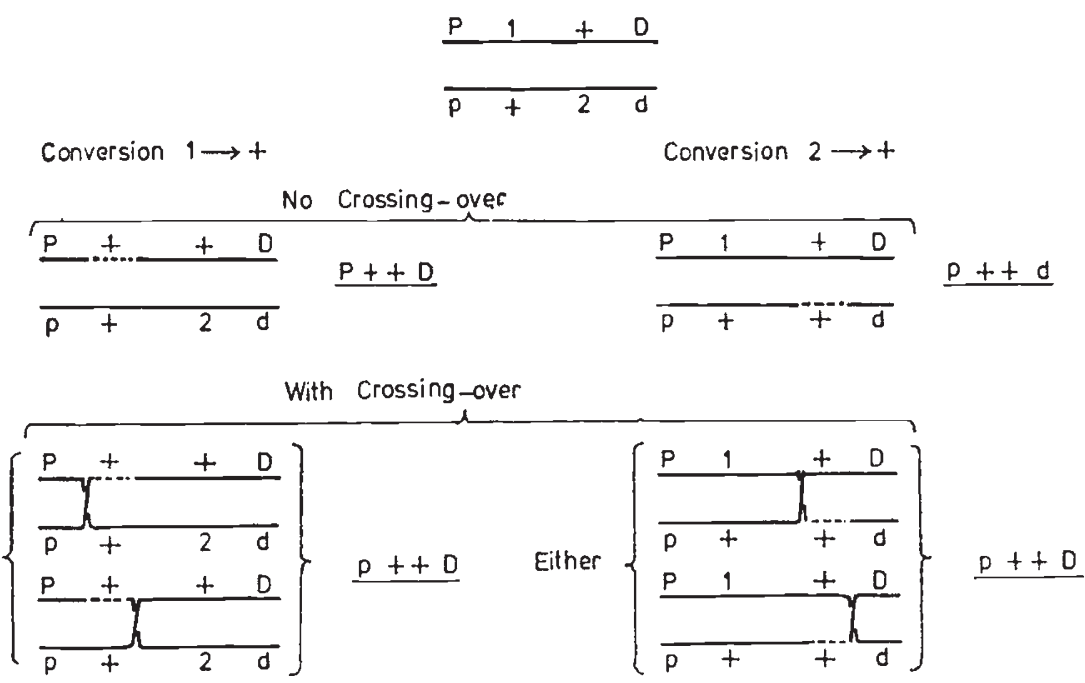

FIG. 1.-To show the flanking marker combinations expected to be found associated with intragenic recombinants due to conversion either with or without adjacent crossing-over.

Although the initial expectation in most analyses of genetic fine-structure in fungi has been that the R1-R2 inequality would be the crucial criterion, it has emerged in a number of cases that at least as strong and consistent an inequality may be seen between $\mathrm{P} 1$ and $\mathrm{P} 2$, and this asymmetry may provide at least as good a criterion of site order as that between R1 and R2. Where both criteria are applicable they give the same gene map (e.g. Smith, 1965). A consistent P1-P2 asymmetry evidently reflects a gradient of conversion frequency within the gene; where $\mathrm{P} 1$ is consistently greater than P2 a more proximal site within the gene tends to be converted in preference to a more distal site, while the reverse is the case where $\mathrm{P} 2>\mathrm{P} 1$.

The purpose of this note is to point out that in some cases the P1-P2 $33 / 1-\mathrm{H} 2$ 
inequality may provide the only means of establishing the gene map (R1 and $\mathrm{R} 2$ being nearly equal) and to discuss briefly the implications of situations of this kind.

A map of the Neurospora crassa am gene (coding for NADP-linked glutamate dehydrogenase) has been made by myself (Fincham, 1967) and more recently by Smyth (1973). There were two main differences between these two studies: firstly, Smyth used stocks which all carried $r e c-3$, a recessive which increases recombination frequency in $a m$ by a factor of approximately 10 (Catcheside, 1968), whereas my stocks were all rec-3+. Secondly, although the same proximal marker was used in both studies (spray, about 5 map units proximal to am), Smyth used a much closer distal marker, histidine-1, 3 map units from am, instead of inositol (inos) which is about 9 map units from am.

The two sets of data are compared in fig. 2 and 3. Fig. 2 is taken from Smyth's paper while fig. 3 is a similar presentation of my own data. In both figures the classification of the flanking marker combinations (P1, P2, R1, R2) is based on the convention that Smyth's suggested orientation of the gene is correct though, as I shall argue, there is some doubt about this.

The two studies show a gratifying agreement in their ordering of the
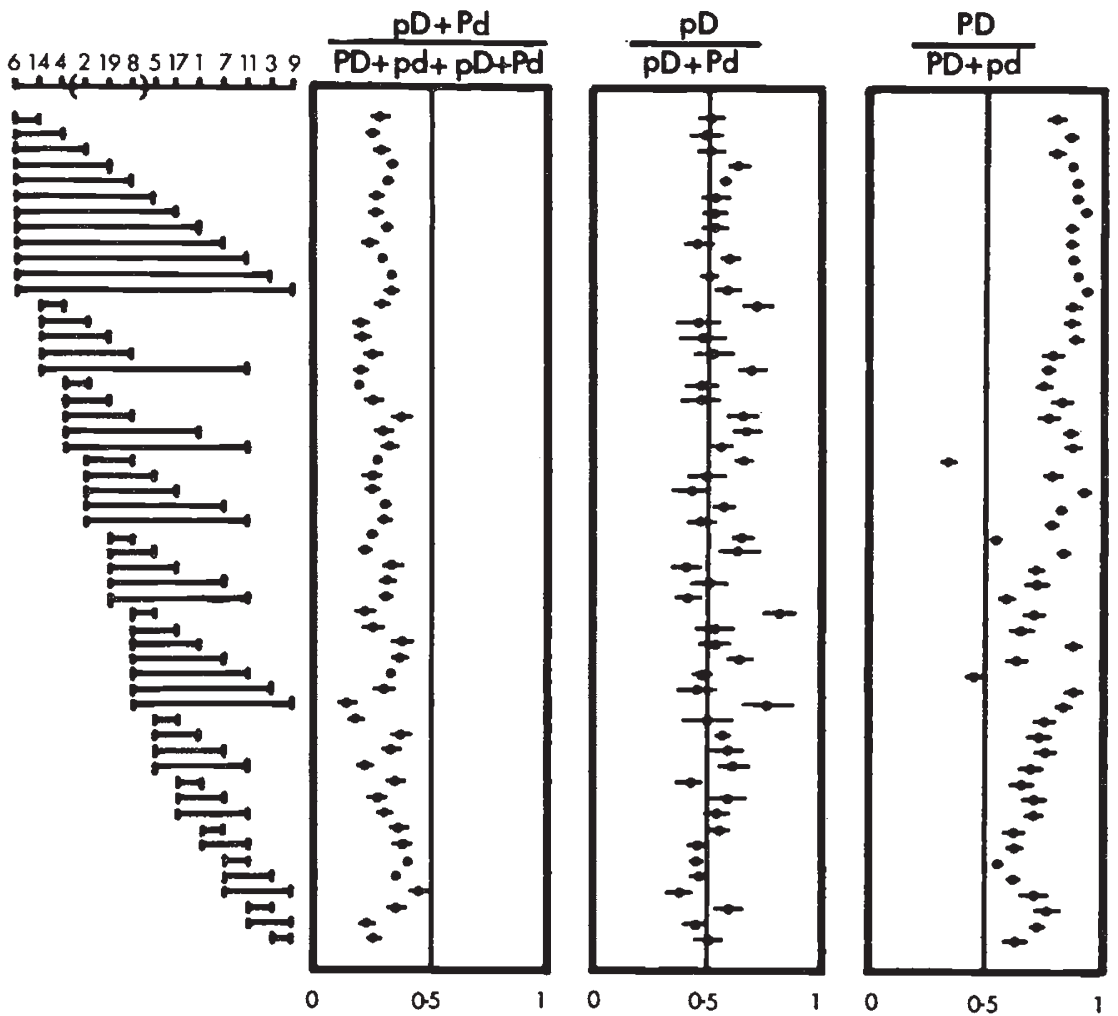

FIG. 2.-Flanking marker distributions among $\mathrm{am}^{+}$recombinants from crosses between am alleles in rec-3 background. The alleles crossed are indicated on the left by horizontal lines between their sites. The order of sites has been chosen primarily to maximise the consistency of the pattern of polarity of conversion (see right-hand panel). Data of D. R. Smyth (1973, reproduced by permission). The horizontal bars indicate standard errors. 
am sites by the criterion of conversion polarity $(\mathrm{P} 1>\mathrm{P} 2)$. This is shown in the right-hand panels of figs. 2 and 3 . The polarity is somewhat stronger in Smyth's data (P1/(P1 + P2) mostly between 0.7 and 0.9$)$ than in mine $(\mathrm{P} 1 /(\mathrm{P} 1+\mathrm{P} 2)$ mostly between 0.6 and $0 \cdot 7)$ almost certainly because of the effect of rec-3 on polarity (Smyth, 1971). Apparent reversals of polarity $(\mathrm{P} 1 /(\mathrm{P} 1+\mathrm{P} 2)<0.5)$ are rare in both studies.
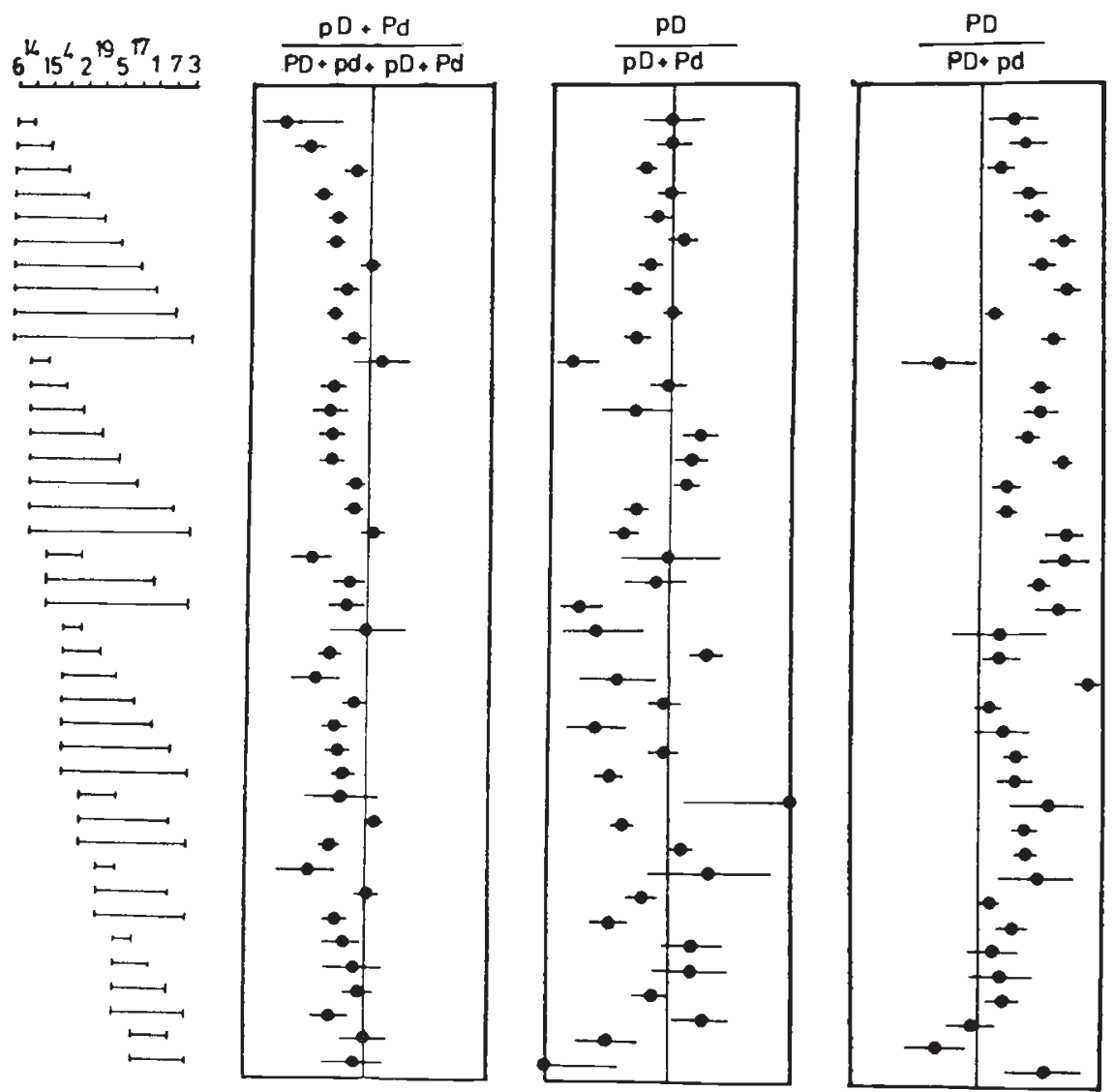

Fig. 3.-As fig. 2, but crosses made in rec-3+ ${ }^{+}$background. Data of Fincham (1967) with additional unpublished results for crosses involving $a m^{15}$ and $a m^{17}$.

The left-hand panel in each figure shows the proportion of $a m^{+}$recombinants from each cross which is recombined for the flanking markers. It will be seen that the two sets of data agree in showing only a minority of $\mathrm{am}^{+}$products in the R1 and R2 classes. The proportion of flanking marker recombinants varies around 33 per cent in Smyth's data and around 40 per cent in mine. Much of the difference between these two numbers is to be accounted for by the wider spacing of the markers in my study (14 units as compared with 8) which doubtless led to a greater inflation of the Rl and $\mathrm{R} 2$ classes by cross-overs formed independently of the recombinational event with the gene. Allowing for such independent cross-overs, intragenic recombination appears to entail crossing-over only about 25 per cent of the time.

Where the two sets of data tend to disagree is in the relative frequencies 
of R1 and R2. In the central panel of fig. 2 it will be seen that there is a somewhat inconsistent but clearly significant tendency for $\mathrm{R} 1>\mathrm{R} 2$, indicating, perhaps, that the left-hand end of the gene map really is proximal (as assumed in the conventions of the figure). Overall, the proportion $\mathrm{R} 1 /(\mathrm{R} 1+\mathrm{R} 2)$ averages about $0 \cdot 6$, though it varies between about $0 \cdot 4$ and 0.7 for different pairs of am alleles, with two higher values. In my data, on the other hand, the balance of values is clearly below $0 \cdot 5$, with a mean of approximately 0.45 and a range of $0 \cdot 2-0 \cdot 7$. This, at face value, would tend to favour the opposite orientation of the gene map, with the left-hand end distal with respect to the centromere. A part of this apparent contradiction between the two sets of data can be attributed to the different flanking markers used. Whereas the proximal interval is about twice as long as the distal interval in the fig. 3 data, the opposite is the case in fig. 2 . Given that the left hand $a m$ site is converted preferentially $(\mathrm{P} 1>\mathrm{P} 2$, purely coincidental crossing-over between $a m$ and the flanking markers will tend to inflate $\mathrm{Rl}$ relative to $R 2$ in the first case and $R 2$ relative to $R 1$ in the second. Calculation shows that this trivial effect cannot account for the whole of the R1-R2 inequality in either set of data nor for the reversal of this inequality between the two. But whatever the reason for the R1-R2 inequality, we seem to be left with no convincing evidence for the orientation of the gene map. Smyth's study, with the closer distal marker and rather greater average R1-R2 inequality, must certainly be given the greater weight but, even so, the variation observed in different crosses and the close approach in many cases to $R_{1}=R_{2}$ leaves considerable room for doubt about the orientation of the gene.

How is the occurrence of such substantial numbers in the R2 class to be explained? One possibility is to suppose that the region within which conversion can occur (by hypothesis a region of hybrid DNA) is long enough to cover all the mutant sites within the gene, and that the sites are far enough apart to undergo independent conversion. In the extreme case, where two sites are always included together in hybrid DNA and are always corrected independently, one would expect $\mathrm{P} 1=\mathrm{P} 2$ and $\mathrm{R} 1=\mathrm{R} 2$. Any departure from these equalities would be due to preferential conversion in one direction rather than the other at one or both sites, and would be unrelated to the orientation of the gene map. There is no reason to think that independent conversion at two heterozygous sites within a gene cannot occur; indeed it has been suggested as a likely explanation for the phenomenon of map expansion (Fincham and Holliday, 1970). But this hypothesis, while it could explain the near-equality, in the am data, of R1 and R2 does not predict the strong and consistent inequality of $\mathrm{P} 1$ and $\mathrm{P} 2$. One escape from the dilemma is to suppose that the events leading to the formation of $\mathrm{R}$ products afford greater opportunities for independent correction (e.g. by the formation of longer stretches of hybrid DNA) than do those leading to $P$ products; this explanation was proposed by Smyth (1971) and was independently suggested to me by Dr Philip Hastings, but it seems a little contrived.

An alternative, which seems to me to be simpler, is to resurrect the idea of negative interference, or clustering of recombinational events (perhaps in regions of especially close synapsis) as suggested by Pritchard (1960). In the $a m$ case the data suggest that recombination within am is due overwhelmingly to conversion without crossing-over directly resulting from the 
same event; but that where such conversion occurs there is about a 25 per cent chance of finding a cross-over as a separately initiated event close by, either to the left or the right with almost equal likelihood. Depending on the statistical distribution of the postulated regions of close synapsis (which, in turn, is likely to be dependent on genetic background) there might be a bias in favour of associated crossing-over on one side of the gene rather than the other. Any such bias would have no relevance to the orientation of the gene map with respect to the chromosome map. The special feature of the am situation would be the low frequency with which conversion is associated with crossing-over in the same primary event; nearly all the extra flanking marker recombination would be due to negative interference. In contrast, the typical Saccharomyces situation seems to be one in which nearly 50 per cent of conversions are directly associated with crossing-over ( $c f$ fig. 1) with relatively little negative interference. Other genes in Neuro ora (i.e. his-5, Smith, 1965; me-2, Murray, 1963) seem to exhibit an inte ediate behaviour, with a direct (though less than 50 per cent) associc ion of conversion with crossing-over (R1>R2) occurring together with, and partially obscured by, negative interference ( $\mathrm{R} 2$ hence a sizeable class).

The question why the direct association of conversion-inducing events with crossing-over should vary between nearly 50 per cent and near zero for different genes or short chromosome segments is an intriguing one. Whatever the answer may be, the use of a small inequality between the $\mathrm{Rl}$ and $\mathrm{R} 2$ classes as a criterion for gene orientation seems very unsafe, especially when the frequency of conversion-associated crossing-over is low.

Acknowledgment.-I am grateful to Dr D. R. Smyth for letting me see his data prior to publication and for constructive discussion and comments on this paper. Thanks are also due to him and to the Australian fournal of Biological Science for permission to use his figure.

\section{REFERENCES}

CATCheside, D. G. 1968. The control of genetic recombination in Neurospora crassa. In Replication and Recombination of the Genetic Material (eds. W. J. Peacock and R. D. Brock). Austr. Acad. Sci., Canberra.

ChOVNick, A., BALlantyne, G. H., AND holm, D. G. 1971. Studies on gene conversion and its relationship to linked exchange in Drosophila melanogaster. Genetics, 69, 179-209.

FINCHAM, J. R. s. 1967. Recombination within the am gene of Neurospora crassa. Genet. Res., Camb., 9, 49-62.

FINCHAM, J. R. S., AND HOLLIDAY, R. 1970. An explanation of fine structure map expansion in terms of excision repair. Molec. Gen. Genetics, 109, 309-322.

FINCHAM, J. R. S., AND DAY, P. R. 1971. Fungal Genetics, pp. 402. Blackwell Scientific Publ., Oxford (see esp. Chapter 8).

HOLlidAy, R. 1968. Genetic recombination in fungi. In Replication and Recombination of the Genetic Material (eds. W. J. Peacock and R. D. Brock). Austr. Acad. Sci., Canberra.

HURST, D. D., FOGEL, S., AND MORTTMER, R. K. 1972. Conversion-associated recombination in yeast. Proc. Nat. Acad. Sci., 69, 101-105.

MURRAY, N. E. 1963. Polarized recombination and fine structure within the $m e-2$ gene of Neurospora crassa. Genetics, 48, 1163-1184.

PRITCHARD, R. H. 1960. Localized negative interference and its bearing on models of gene recombination. Genet. Res., Camb., 1, 1-24.

SMTTH, B. R. 1965. Interallelic recombination at the histidine-5 locus in Neurospora crassa. Heredity, 20, 257-276.

SMYTH, D. R. 1971. Effect of rec-3 on polarity of recombination in the amination-1 locus of Neurospora crassa. Austr. F. Biol. Sci., 24, 97-106.

SMYTH, D. R. 1973. A new map of the amination-1 locus of Neurospora crassa, and the effect of the recombination-3 gene. Austr. F. Biol. Sci., 26, 1355-1370. 